\title{
Liver related complications in unresectable disease after portal vein embolization
}

\author{
Floor Huisman ${ }^{1}$, Kasia P. Cieslak ${ }^{1}$, Krijn P. van Lienden ${ }^{2}$, Roelof J. Bennink ${ }^{3}$, Thomas M. van Gulik ${ }^{1}$ \\ ${ }^{1}$ Department of Surgery, ${ }^{2}$ Department of Radiology, ${ }^{3}$ Department of Nuclear Medicine, Academic Medical Center, Amsterdam, The Netherlands \\ Contributions: (I) Conception and design: KP van Lienden, RJ Bennink, TM van Gulik; (II) Administrative support: F Huisman, KP Cieslak; \\ (III) Provision of study material or patients: All authors; (IV) Collection and assembly of data: F Huisman, KP Cieslak; (V) Data analysis and \\ interpretation: All authors; (VI) Manuscript writing: All authors; (VII) Final approval of manuscript: All authors. \\ Correspondence to: Prof. Dr. Thomas M. van Gulik, MD. Department of Surgery, Academic Medical Center, IWO IA.1-119, 1100 DD Amsterdam, \\ the Netherlands. Email: t.m.vangulik@amc.uva.nl.
}

\begin{abstract}
Background: Portal vein embolization (PVE) is used preoperatively in patients to increase future remnant liver volume (FRLV). Unfortunately, some patients are found to be unresectable at exploration due to tumor progression or new lesions. The aim of this study is to evaluate the long-term effects of PVE in the embolized liver lobe when left unresected.

Methods: Of 85 patients who underwent right PVE, 16 (19\%) were unresectable (PVE-group). These patients were compared with 48 randomly matched patients from a pool of 75 unresectable patients who had not undergone PVE. Primary outcome parameter was occurrence of infectious complications (liver abscesses) on follow-up imaging of the liver. The long-term volumetric changes of the hypertrophy/atrophy complex were assessed as secondary outcome parameter.

Results: Five of 16 (31\%) patients in PVE-group developed an abscess vs. 4 (8\%) patients in non-PVE group ( $\mathrm{P}=0.022)$. The volume distribution of left and right liver lobes (hypertrophy-atrophy rate) increased from 26\%:74\% before embolization to 36\%:64\% three weeks after PVE and to 51\%:49\% six months after PVE.

Conclusions: Persistence of embolized liver lobe in unresectable patients after PVE resulted in abscesses in $31 \%$. This observation calls for developing reversible embolization techniques using absorbable materials in patients with uncertain resectability.
\end{abstract}

Keywords: Portal vein embolization (PVE); liver surgery; complications; liver abscesses

Submitted Oct 28, 2016. Accepted for publication Jan 13, 2017.

doi: $10.21037 / \mathrm{hbsn} .2017 .02 .03$

View this article at: http://dx.doi.org/10.21037/hbsn.2017.02.03

\section{Introduction}

Liver resection is the main curative therapy for primary or metastatic liver tumors. The volume of the liver that remains after resection (future remnant liver, FRL) should be at least $25-30 \%$ of the total liver volume (TLV) based on computed tomography (CT) volumetric studies. An insufficient FRL volume (FRLV) is associated with increased rates of postoperative liver failure and liver failure related mortality (1). In case of compromised liver tissue, e.g., due to steatosis, cirrhosis or recent chemotherapy, FRLV of at least $40 \%$ is preferred (2). However, in patients with extensive disease who require major liver surgery (resection of 3 or more Couinaud segments), the FRLV is often lower than the accepted cut-off values for safe resection.

Portal vein embolization (PVE) is an accepted method to preoperatively increase FRLV. This technique uses the unique capacity of the liver to regenerate. As a result of selective portal vein occlusion, atrophy of the embolized liver lobes occurs with a concomitant hypertrophy response of the non-embolized liver lobes. Permanent occlusion of the portal vein is usually achieved by using non-absorbable embolization materials, such as polyvinyl alcohol particles 
Table 1 Baseline characteristics of patients (March 2005 to August 2013) who had undergone right portal vein embolization (PVE group) and the matched control group who had not undergone PVE (non-PVE group) before being defined as unresectable

\begin{tabular}{|c|c|c|c|}
\hline Patients & PVE group, $n=16$ & Non-PVE group, $n=48$ & $P$ value \\
\hline Age in years, mean $\pm S D$ & $66 \pm 6.9$ & $64 \pm 11.3$ & 0.661 \\
\hline BMI, median (IQR 25-75) & 24 [22-27] & 23 [20-26] & 0.201 \\
\hline Exploration by laparotomy (\%) & $11(68.8)$ & $24(50.0)$ & 0.244 \\
\hline \multicolumn{4}{|l|}{ Diagnosis (\%) } \\
\hline CRLM & $10(62.5)$ & $30(62.5)$ & \\
\hline $\mathrm{PHC}$ & $16(37.5)$ & $18(37.5)$ & \\
\hline Palliative chemotherapy* (\%) & $9(56.0)$ & $26(54.2)$ & 0.885 \\
\hline
\end{tabular}

*, given after unresectability was defined. SD, standard deviation; IQR, interquartile range; CRLM, colorectal liver metastases; PHC, perihilar cholangiocarcinoma.

with coils (PVAc), n-butyl cyanoacrylate (NBCA), fibrin glue, ethanol or combinations of these. Unfortunately, recent studies have shown that approximately $20 \%$ of the originally planned liver resections after PVE were cancelled, because of insufficient hypertrophy response of the FRL, intrahepatic tumor progression, new hepatic metastases or extra-hepatic tumor spread (3). This is consistent with our own experience where $19 \%$ of the patients after having undergone PVE [2005-2013] were found to be unresectable. Remarkably, we have observed infectious complications in the embolized liver lobe in a number of patients who had undergone PVE, but eventually did not undergo resection. The long-term effects of permanent occlusion of portal vein on the embolized, atrophied liver lobe have not been described in literature so far. Hence, the aim of this study is to evaluate the long-term effects of PVE in the embolized liver lobe when left unresected.

\section{Methods}

\section{Patients}

From March 2005 to August 2013, 85 patients underwent PVE of the right portal system. Sixteen out of the 85 (19\%) patients were found to be unresectable (PVE group). Of these patients, 10/16 (62.5\%) were diagnosed with colorectal liver metastases (CRLM) and 6/16 (37.5\%) with perihilar cholangiocarcinoma (PHC). These patients were compared with 48 randomly matched patients with CRLM and PHC (CRLM-PHC ratio of 3:1) from a pool of 75 patients who did not undergo preoperative PVE and were found unresectable during the preoperative workup or during laparotomy (non-PVE group) in the same period. We used IBM SPSS Statistics (SPSS for Windows; SPSS, Chicago, Illinois, USA) to create a random sample. The tumor load (number and size of metastasis) in patients diagnosed with CRLM $(n=30)$ was equally distributed in the PVE-group and the non-PVE group. Furthermore, patients diagnosed with $\mathrm{PHC}$ in both groups $(\mathrm{n}=10)$ were classified as Klatskin type 3 or 4 . The baseline characteristics of patients in the PVE and non-PVE group are shown in Table 1.

\section{Preoperative work-up in the PVE group}

All included patients were evaluated by a multidisciplinary team, where treatment was defined by consensus. PVE was indicated when the FRL was $<25-30 \%$ of TLV for patients with normal liver parenchyma, and $<35-40 \%$ in case of underlying liver disease (e.g., steatosis, cirrhosis, recent chemotherapy). In addition to volumetric measurements, the functional capacity of FRL was assessed using technetium-99m ${ }^{99 \mathrm{~m}} \mathrm{Tc}$ )-mebrofenin hepatobiliary scintigraphy (HBS) as described previously (4). PVE was indicated in case HBS showed FRL function below the validated cut-off value $\left(2.69 \% / \mathrm{min} / \mathrm{m}^{2}\right)(5)$.

\section{Volumetric assessment of the liver}

Preoperative CT imaging and volumetric assessment of the FRL were performed in all patients. CT-volumetry was 

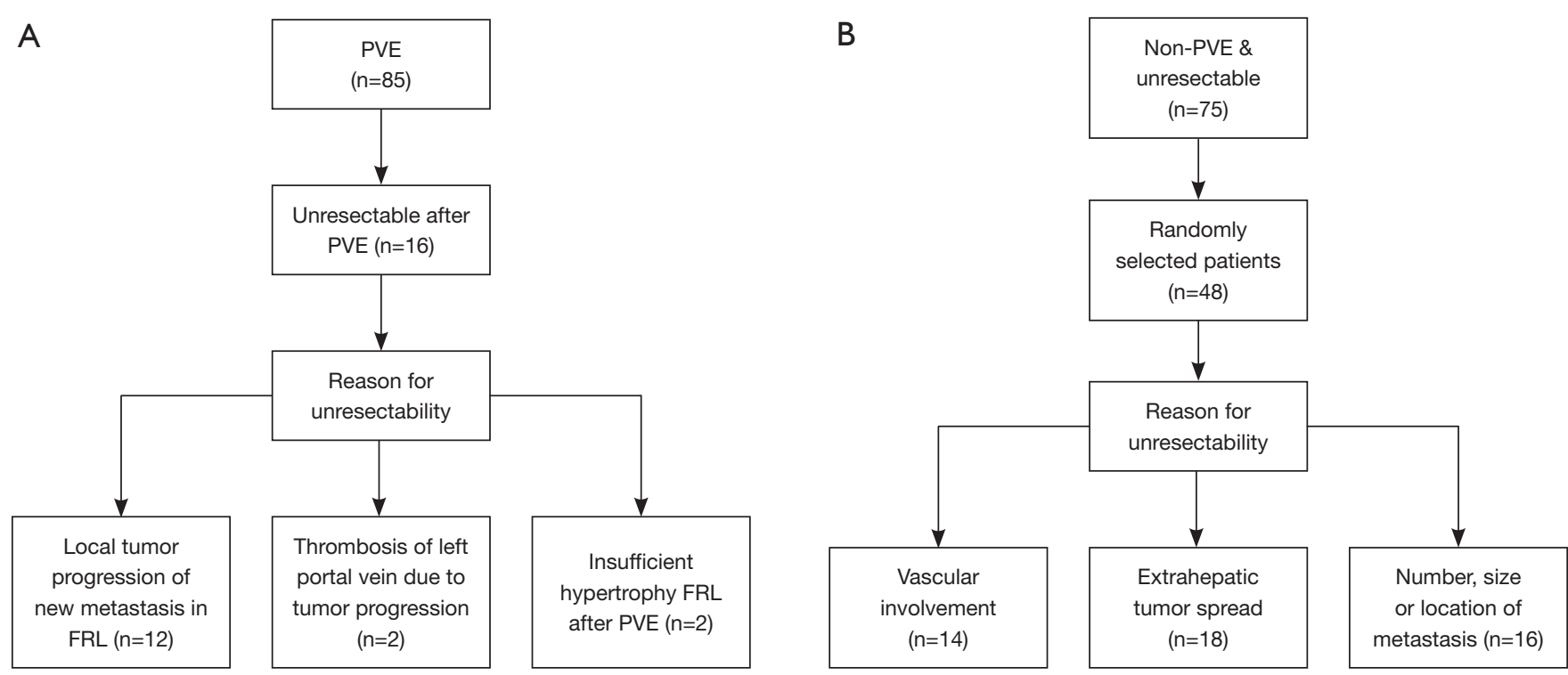

Figure 1 Reasons for unresectability in patients who underwent portal vein embolization (PVE) (A) and in patients of the non-PVE group (B).

performed before and three weeks after PVE. The portalvenous phase of the CT-scans was used for the volumetry. $3 \mathrm{D}$ reconstructions of the liver were made using $5 \mathrm{~mm}$ thick axial slices. Portal and hepatic veins were used as landmarks for segmental division. The circumferences of total liver, tumor and FRL were outlined manually. Integrated software (Mx-View 3.52, Philips Medical Systems) was used to calculate TLV, tumor volume (TV) and FRL volume (FRLV). The percentage FRLV was calculated using the following formula: $[\mathrm{FRLV} /(\mathrm{TLV}-\mathrm{TV})] \times 100$. Tumor progression was documented when detected after PVE.

When unresectability was concluded after PVE (Figure 1A), follow-up imaging (CT-scan or ultrasound) of the liver was performed in all 16 patients in the PVE group. In $11(68.8 \%)$ of these patients, a follow-up CTscan was available after a mean interval of $6 \pm 2$ months following PVE. Possible recanalization of the occluded portal venous system was evaluated using the portal phase images. Volumetric studies were performed to measure the long-term hypertrophy-atrophy rates of embolized and non-embolized lobes, respectively. Three patients had died without follow-up scans and two patients had only followup ultrasound examinations; therefore, no volumetric data were available of these patients. One patient diagnosed with PHC underwent follow-up HBS at 20 months after PVE to evaluate long-term changes in function of the embolized and non-embolized liver lobes.

\section{HBS evaluation of liver function}

Liver function was evaluated quantitatively using HBS before and 3 weeks after PVE as published before $(4,5)$. A cut-off value of at least $2.69 \% / \mathrm{min} / \mathrm{m}^{2}$ was considered compatible with sufficient residual liver function. Data were processed on a Hermes workstation (Hermes Medical Solutions, Sweden).

\section{Unresectable disease and follow-up in non-PVE patients}

Patients in the control group were deemed unresectable by a multidisciplinary team because of number and location of lesions as evidenced by radiological findings, or were found to be unresectable during laparotomy (Figure 1B). Followup imaging was performed to evaluate the response to chemotherapy after unresectability was defined.

\section{Chemotherapy}

Palliative chemotherapy was offered to all patients who were considered unresectable. Chemotherapeutic regimens were scheduled according to the local protocols, but if needed, adapted to the individual patient. The combination of oxaliplatin and capecitabine with or without bevacizumab was given in most cases (CRLM). Some patients received capecitabine, irinotecan, panitumumab, or oxaliplatin with 
Table 2 Volumetric measurements of the total liver, FRLV and RLV before and 3 weeks after PVE in all 16 patients in the PVE group

\begin{tabular}{|c|c|c|c|}
\hline CT-volumetry & Before PVE & After PVE & $P$ value \\
\hline $\mathrm{FRLV}$ in $\mathrm{mL}$, mean $\pm \mathrm{SD}$ & $519 \pm 167$ & $699 \pm 209$ & $<0.001$ \\
\hline $\mathrm{RLV}$ in $\mathrm{mL}$, mean $\pm \mathrm{SD}$ & $1,405 \pm 324$ & $1,198 \pm 543$ & 0.005 \\
\hline$\%-F R L$, mean \pm SD & $28 \pm 7$ & $37 \pm 6$ & $<0.001$ \\
\hline
\end{tabular}

PVE, portal vein embolization; FRLV, future remnant liver volume; RLV, right liver volume; SD, standard deviation; \%-FRL, future remnant volume as percentage of the total liver volume; \%-RLV, right liver volume as percentage of the total liver volume.

5 -fluorouracil/leucovorin. The chemotherapy regime for cholangiocarcinoma consisted of a combination of gemcitabine and cisplatin (6). Due to the large variation of the received chemotherapy regimens, we only recorded whether patients received palliative chemotherapy or not.

\section{Study outcomes}

The primary outcome parameter of this study was the occurrence of infectious complications in unresected patients, defined as the finding of liver abscesses on followup imaging of the liver. All patients received follow-up CT scans. When a liver abscess was clinically suspected, an ultrasound and subsequent CT were made. Most of the patients presented with the clinical picture of an abscess, i.e., fever and elevated CRP.

The secondary outcome parameter was volumetric changes of the embolized and non-embolized liver lobes reflecting long-term hypertrophy/atrophy rates after PVE. The volume distribution was defined as the rate between the percentage FRLV of the TLV and the percentage right liver volume of the TLV, i.e., \%FRLV:\% RLV. In one patient, we were able to assess long-term changes in the hepatic uptake function of the embolized and non-embolized lobes using HBS.

\section{Statistical analysis}

The data were analysed by statistical software (SPSS for Windows; SPSS, Chicago, Illinois, USA) and GraphPad Prism (Graph-pad, San Diego, CA, USA). The nonparametric Mann Whitney $U$ test was used for comparing data that was not normally distributed between the PVEgroup and the non-PVE group. Survival curves were generated by the Kaplan-Meier method. All statistical tests were two-tailed, and differences were considered significant at a $\mathrm{P}$ value of $\leq 0.05$.

\section{Results}

\section{PVE}

PVE of the right portal venous system was successfully performed in all 16 unresectable patients in the PVE-group. No procedure related complications were noted. The median time interval between PVE and surgical exploration was 36 days (IQR 32-58 days). The mean \%FRLV measured by CT volumetry before PVE was $28 \% \pm 7 \%$. Three to 4 weeks (mean $21 \pm 3$ days) after PVE, the \%FRLV had increased to $37 \% \pm 6 \%(\mathrm{P}<0.001)$ (Table 2). HBS showed a mean ${ }^{99} \mathrm{Tc}$-mebrofenin uptake rate in the FRL of $2.04 \% \pm 0.95 \% / \mathrm{min} / \mathrm{m}^{2}$ before PVE. Three to 4 weeks (mean $22 \pm 2$ days) after PVE, the mean ${ }^{99} \mathrm{Tc}$-mebrofenin uptake rate in the FRL had increased to $3.37 \% \pm 1.13 \% / \mathrm{min} / \mathrm{m}^{2}$, $\mathrm{P}=0.001$. The mean hepatic mebrofenin uptake rate of the atrophic, right liver lobes was $3.90 \% \pm 1.75 \% / \mathrm{min} / \mathrm{m}^{2}$ before embolization and $3.03 \% \pm 1.17 \% / \mathrm{min} / \mathrm{m}^{2}$ after PVE, $\mathrm{P}=0.010$. There were no differences in the mean uptake of the right liver lobes after embolization between patients who developed liver abscesses and patients in whom no abscesses were seen after the procedure, $\mathrm{P}=0.699$.

\section{Reasons for unresectability}

In the PVE-group, 5 patients out of the 16 (31\%) did not undergo exploration due to evident tumor progression on the follow-up CT-scans 3 weeks post PVE. The remaining 11 patients were found unresectable during exploration. In the non-PVE group, 24 out of the 48 (50.0\%) patients were found to be unresectable at exploration while the remaining 
24 patients were considered unresectable based on preoperative imaging. Baseline characteristics of the PVE and the non-PVE patients are shown in Table 1. The causes of unresectability in both groups are shown in Figure 1 .

\section{Follow-up imaging and occurrence of PVE related long- term complications}

Recanalization of the occluded portal venous system did not occur in any of the 16 patients in the PVE group. Five out of the $16(31 \%)$ patients in the PVE group developed

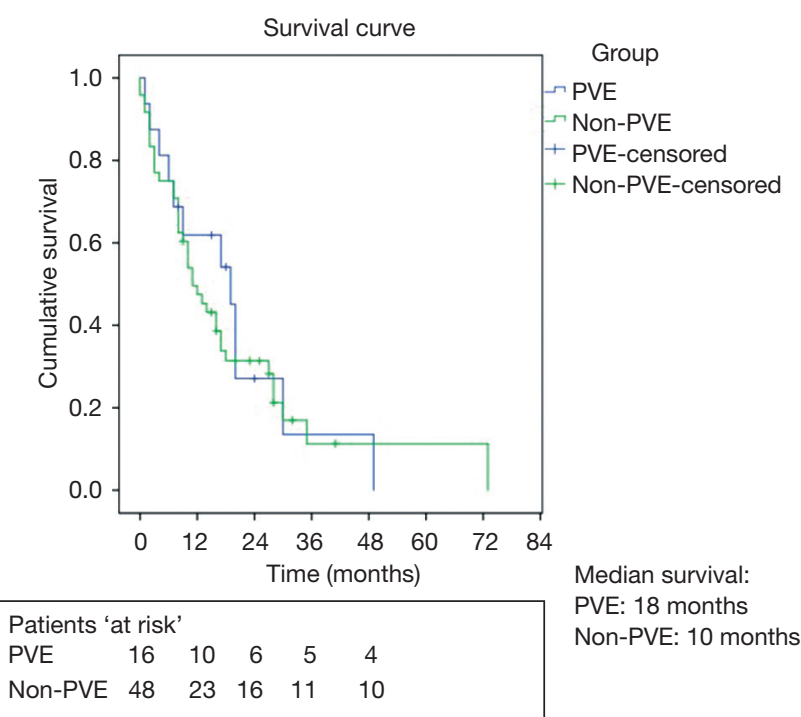

Figure 2 Cumulative survival of patients who underwent portal vein embolization (PVE) and were unresectable and patients who did not undergo PVE. one or more abscess diagnosed on CT scan or ultrasound vs. 4 (8\%) patients in the non-PVE group, $\mathrm{P}=0.022$. Of the 5 patients who had developed an abscess in the PVE group, $3 / 5(60 \%)$ were diagnosed with PHC and $2 / 5(40 \%)$ with CRLM. In the non-PVE group, $1 / 4$ (25\%) patient with abscesses was diagnosed with CRLM and the remaining 3 (75\%) patients with PHC.

In the PVE-group, the median survival of unresectable patients was 18 months (IQR 6-20 months), including the 4 patients with liver abscesses. Median survival in the control group was 10 months (IQR 7-17 months), which was not significantly different from the PVE-group $(\mathrm{P}=0.66$, Figure 2).

\section{The long-term hypertrophy/atrophy rates}

After a mean time of $6 \pm 2$ months, volumetric measurements were performed using follow-up CT-scans in 11/16 (68.8\%) patients who had undergone PVE. In this subgroup, the median \%FRLV before PVE was $26 \% \pm 7 \%$ and after PVE had increased to $36 \% \pm 6 \%, \mathrm{P}<0.001$. Ongoing hypertrophy was seen 6 months after PVE as the \%FRLV had increased to $51 \% \pm 13 \%(\mathrm{P}<0.001$, Table 3$)$.

The volume distribution of the left and right liver lobes (hypertrophy-atrophy rate) increased from 26\%:74\% before embolization to $36 \%: 64 \%$ three weeks after PVE and to 51\%:49\% six months after PVE (Figure 3).

As mentioned in the methods section, one patient (65 years, female) with biopsy proven PHC underwent functional assessment of the FRL during follow-up with HBS. She underwent HBS scans before PVE and 3 weeks, 17 and 20 months after PVE, respectively (Table 4). The

Table 3 Volumetric measurements of the 11 patients in the PVE group in whom follow-up CT imaging was performed

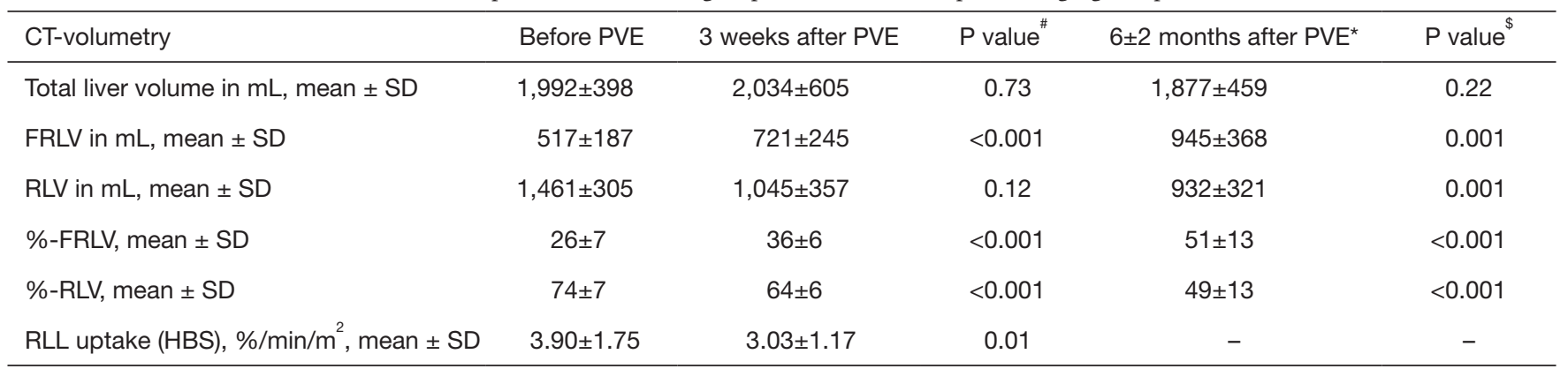

\#, comparison of values before vs. 3 weeks after PVE; *, after a median time of $6 \pm 2$ months; ${ }^{\$}$, comparison of values 3 weeks after PVE vs. 6 months. PVE, portal vein embolization; FRLV, future remnant liver volume; RLV, right liver volume; RLL, right liver lobes; SD, standard deviation. 

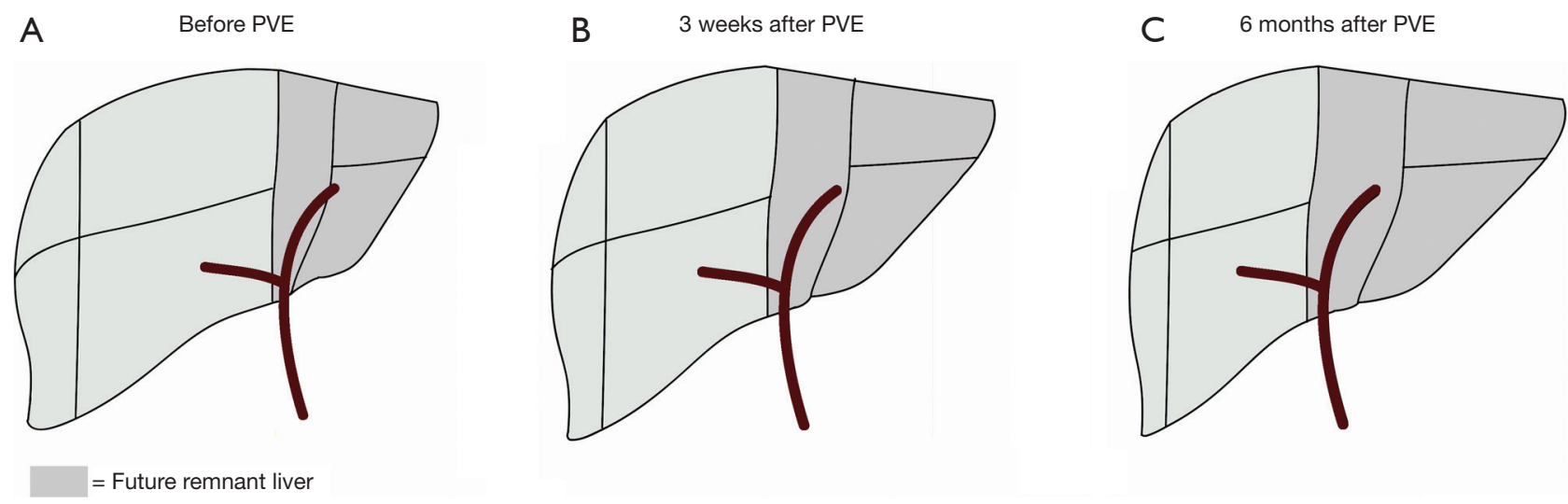

Figure 3 Volume distribution of the future remnant liver (FRL) and right (embolized) liver. Median \%FRL increased from $26 \% \pm 7 \%$ pre portal vein embolization $(\mathrm{PVE})$ to $36 \% \pm 6 \%(\mathrm{P}<0.001)$ at 3 weeks after $\mathrm{PVE}$ and to $51 \% \pm 13 \%(\mathrm{P}<0.001) 6$ months after $\mathrm{PVE}$.

Table 4 Follow-up HBS in one unresectable patient who underwent PVE

\begin{tabular}{lcccc}
\hline CT-volumetry & Before PVE & 3 weeks after PVE & 17 months after PVE* & 20 months after PVE \\
\hline Total liver uptake, \%/min & 15.5 & 15.1 & 4.9 & 42 \\
\% FRL of total liver uptake & 24 & 46 & 58 & 68 \\
\% embolized lobes of total liver uptake & 76 & 54 & 3.3 & 3.9 \\
FRL uptake, \%/min/m & 1.8 & 3.4 & 1.3 & 1.1 \\
Embolized liver lobes uptake, \%/min/m & 5.8 & 4.1 & & 2.3 \\
\hline
\end{tabular}

*, cholangitis due to insufficient biliary drainage during follow-up HBS at 17 months after PVE showing a relevant decrease in FRL uptake rate. PVE, portal vein embolization; FRL, future remnant liver; HBS, Hepatobiliary-scintigraphy (HBS) using ${ }^{99 m}$ Tc-mebrofenin.

measured uptake function of the embolized, atrophied liver lobe had decreased considerably after 20 months compared to the initial function, i.e., from $5.8 \% / \mathrm{min} / \mathrm{m}^{2}$ to $1.1 \% / \mathrm{min} / \mathrm{m}^{2}$. Figure 4 illustrates the changes and distribution of the hepatic uptake function in the embolized and non-embolized liver lobes.

\section{Discussion}

PVE is a safe and frequently used method to induce hypertrophy of the FRL. Owing to PVE, more patients have become eligible for curative liver resection with less postoperative complications (6). Unfortunately, in approximately $20 \%$ of the cases, patients are found to be unresectable in the time between PVE and operation due to tumor progression or the occurrence of extra-hepatic metastases (7).

Tumor growth of microscopic and macroscopic liver lesions after PVE has been increasingly reported in literature, and can be considered a short-term complication of the procedure (8). The long-term effects of the persisting embolized lobe after PVE in unresectable patients are largely unknown. The atrophy-hypertrophy complex stabilizes in time while providing sufficient liver function and complications of the atrophied liver lobe have not been reported so far (9). We assumed that, especially in patients with $\mathrm{PHC}$, troublesome septic complications may occur due to infected and incompletely drained bile ducts while the hilar tumor progresses and continues to occlude (sub)segmental bile ducts. We have shown in this study that unresected patients with not only PHC but also with CRLM who had undergone PVE are more prone to develop abscesses compared to unresected patients with the same diagnoses and a patent portal vein of the right liver. In case of CRLM, the etiology of abscess formation is less clear. Possibly parenchymal damage following extensive chemotherapy and/or local ischemia due to tumor progression plays a role. Although the occurrence of 

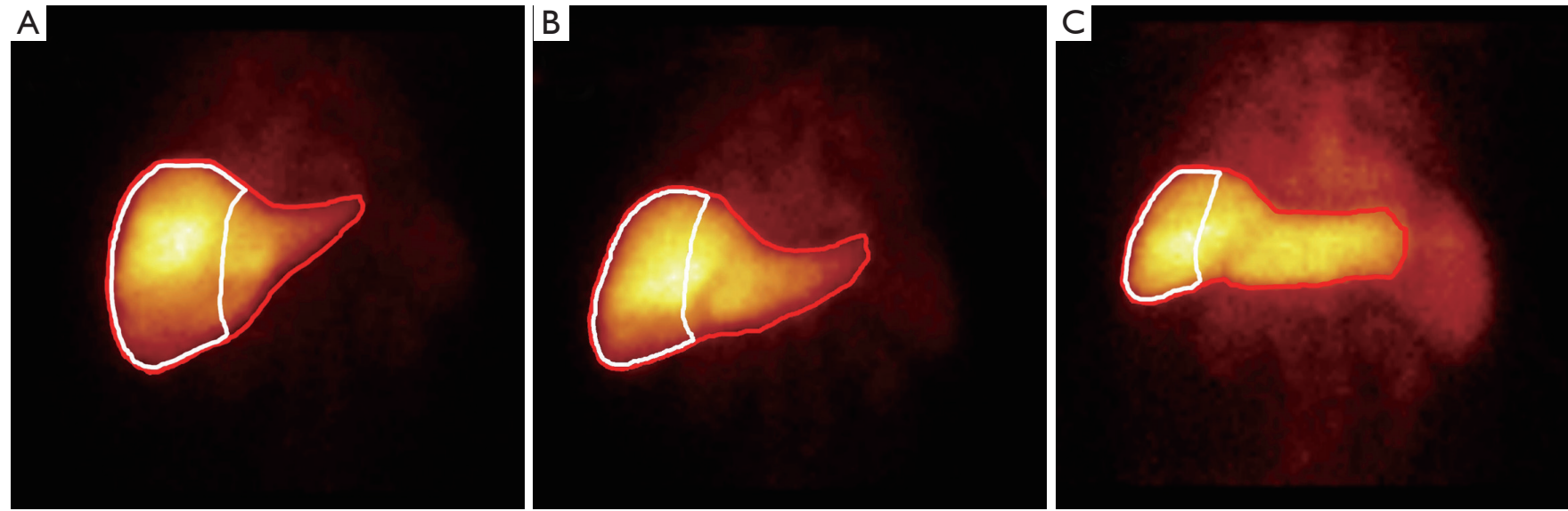

Figure 4 Hepatobiliary scintigraphy (HBS) imaging performed before (A), 3 weeks (B) and 20 months (C) after right portal vein embolization in a patient diagnosed with perihilar cholangiocarcinoma (female; 64 years). The red line delineates the total liver and the white line delineates the right (embolized) liver segments.

abscesses differed significantly between unresectable PVE and non-PVE patients, this did not influence long-term survival as the survival between the groups was comparable. However, the consequences of intrahepatic abscesses impose considerable burden in patients who are undergoing palliative treatment and already have a limited quality of life. To avoid the abovementioned complications, patients who require PVE probably could benefit from the use of absorbable embolization materials, provided that these materials induce an adequate hypertrophy response of the FRL after a predictable absorption period.

Lainas et al. (10) were the first to describe PVE with an absorbable embolization material. Using gelatin sponge powder, the authors embolized the right liver lobes of 9 monkeys and reported a $43 \%$ volume increase of the FRL after 1 month. Recanalization of the embolized portal system was seen 12-16 days after PVE in all animals indicating that the gelatin sponge had been absorbed. van den Esschert et al. (11) compared the hypertrophy response after PVE in rabbits using two absorbable embolization materials. A recent study published by Olthof et al. (12) found an adequate hypertrophy response using a combination of fibrin glue and aprotinin as embolization material showing $80 \%$ recanalization after 42 days in a rabbit model of PVE. The use of absorbable embolization materials therefore seems a promising approach in preventing the long-term complications of permanent embolization. Reversible embolization techniques and their clinical application await validation in further studies.
We evaluated the role of hepatic uptake-function of the embolized liver lobes, as measured with HBS, and its possible contribution to the development of liver abscesses after PVE. The mean uptake function of the embolized liver lobes 3 weeks after PVE decreased significantly in this series. One of the patients with a remarkable long-term survival despite unresectable disease, received several HBS scans during the follow-up period after PVE, showing a decrease of total liver function and relative function of the embolized liver segments in 20 months. This observation corroborates the notion that in case of unresectability after PVE, an absorbable embolization material potentially prevents functional deterioration of the embolized liver lobes and additionally prevents abscess formation in these lobes.

This study has a few limitations. Firstly, we compared initially resectable patients after PVE with unresectable patients without PVE, possibly introducing a selection bias. However, this bias is difficult to avoid since in order to evaluate undesirable long-term complications after PVE we need to compare with patients who did not undergo the procedure. Secondly, we disposed of a relatively small sample size for the PVE group. Notwithstanding these limitations, this is the first report on long-term complications after PVE in unresectable patients.

In conclusion, persistence of the embolized liver lobe in unresectable patients after PVE resulted in abscesses in $31 \%$. This observation calls for developing reversible embolization techniques of the portal vein using absorbable materials in patients with uncertain resectability. 


\section{Acknowledgements}

The authors would like to thank Evelien Huisman for her contribution in graphic design and Martin Poulus from the Department of Radiology for measuring the future remnant liver volumes.

\section{Footnote}

Conflicts of Interest: The authors have no conflicts of interest to declare, as none of the listed authors has a relationship with E-AHPBA that could influence the results of this study or received funding from this organization.

Ethical Statement: Because this was a retrospective study in which patients received standard procedures (i.e., PVE or not), approval was waived by our institutional Medical Ethical Review Board.

\section{References}

1. Kishi Y, Abdalla EK, Chun YS, et al. Three hundred and one consecutive extended right hepatectomies: evaluation of outcome based on systematic liver volumetry. Ann Surg 2009;250:540-8.

2. van Gulik TM, van den Esschert JW, de Graaf W, et al. Controversies in the use of portal vein embolization. Dig Surg 2008;25:436-44.

3. van Lienden KP, van den Esschert JW, de Graaf W, et al. Portal vein embolization before liver resection: a systematic review. Cardiovasc Intervent Radiol 2013;36:25-34.

4. de Graaf W, van Lienden KP, Dinant S, et al. Assessment

Cite this article as: Huisman F, Cieslak KP, van Lienden KP, Bennink RJ, van Gulik TM. Liver related complications in unresectable disease after portal vein embolization. HepatoBiliary Surg Nutr 2017;6(6):379-386. doi: 10.21037/ hbsn.2017.02.03 of future remnant liver function using hepatobiliary scintigraphy in patients undergoing major liver resection. J Gastrointest Surg 2010;14:369-78.

5. de Graaf W, van Lienden KP, van den Esschert JW, et al. Increase in future remnant liver function after preoperative portal vein embolization. Br J Surg 2011;98:825-34.

6. May BJ, Talenfeld AD, Madoff DC. Update on portal vein embolization: evidence-based outcomes, controversies, and novel strategies. J Vasc Interv Radiol 2013;24:241-54.

7. Pommier R, Ronot M, Cauchy F, et al. Colorectal liver metastases growth in the embolized and non-embolized liver after portal vein embolization: influence of initial response to induction chemotherapy. Ann Surg Oncol 2014;21:3077-83.

8. Hoekstra LT, van Lienden KP, Doets A, et al. Tumor progression after preoperative portal vein embolization. Ann Surg 2012;256:812-7; discussion 817-8.

9. Mueller L, Grotelueschen R, Meyer J, et al. Sustained function in atrophying liver tissue after portal branch ligation in the rat. J Surg Res 2003;114:146-55.

10. Lainas P, Boudechiche L, Osorio A, et al. Liver regeneration and recanalization time course following reversible portal vein embolization. J Hepatol 2008;49:354-62.

11. van den Esschert JW, van Lienden KP, Alles LK, et al. Liver regeneration after portal vein embolization using absorbable and permanent embolization materials in a rabbit model. Ann Surg 2012;255:311-8.

12. Olthof PB, Huisman F, van Golen RF, et al. Use of an absorbable embolization material for reversible portal vein embolization in an experimental model. Br J Surg 2016;103:1306-15. 